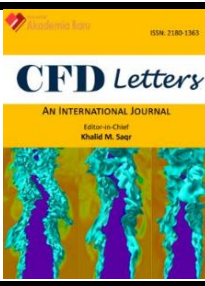

\title{
A Computational Study of a Passive Flow Device in a Mechanical Heart Valve for the Anatomic Aorta and the Axisymmetric Aorta
}

\author{
Nursyaira Mohd Salleh ${ }^{1}$, Mohamad Shukri Zakaria ${ }^{1,2, *}$, Mohd Juzaila Abd Latif $^{1,3}$, Adi Azriff Basri ${ }^{4}$ \\ Fakulti Kejuruteraan Mekanikal, Universiti Teknikal Malaysia Melaka, Hang Tuah Jaya, 76100 Durian Tunggal, Melaka, Malaysia \\ Centre for Advanced Research on Energy, Universiti Teknikal Malaysia Melaka, Hang Tuah Jaya, 76100 Durian Tunggal, Melaka, Malaysia \\ Advanced Manufacturing Centre, Universiti Teknikal Malaysia Melaka, Hang Tuah Jaya, 76100 Durian Tunggal, Melaka, Malaysia \\ Department of Aerospace Engineering, Faculty of Engineering, Universiti Putra Malaysia, 43400 Serdang, Selangor, Malaysia
}

ARTICLE INFO ABSTRACT

\section{Article history:}

Received 10 December 2020

Received in revised form 5 April 2021

Accepted 6 April 2021

Available online 30 April 2021
Keywords:

Pulsatile flow; thrombosis; CFD

simulation; BMHV; vortex generator

\begin{abstract}
Artificial heart valves for replacing diseased indigenous heart valves were widely used. The treatment of certain types of heart disease requires mechanical valves to be implanted operatively. Healthy cardiac valves are essential to proper cardiac function. The current study presents an investigation of the pulsatile blood flow through a bileaflet mechanical heart valve (BMHV) with a vortex generator (VG) in fully open position. A St. Jude Medical Regent valve with a diameter of $23 \mathrm{~mm}$ was used to mount triangular VGs as a means of improving pressure gradients and reducing turbulence. The anatomic aorta and axisymmetric aorta was computed by large eddy simulation (LES) approached. The implications for both models with VGs were observed in terms of velocity magnitude, vortices and wall shear stress. The results suggested that the anatomic aorta is prone to develop more blood clotting at the leading edge of the leaflets with $2.03 \mathrm{~m} / \mathrm{s}$. Furthermore, the anatomic aorta produces higher wall shear stress with 69Pa, which possibly contributes to a high risk of thrombosis.
\end{abstract}

\section{Introduction}

The past decades have witnessed considerable research into heart valve replacement such as the mechanical heart valve (MHV) and the bioprosthetic heart valve (BHV). Among these replacements are bileaflet mechanical heart valves (BMHV) because of their durability and long life span, as they are usually made from pyrolytic-carbon [1]. The design of BMHVs tends to lead to various complications including the risk of thrombosis (blood clotting) and hemorrhage [2]. These complications are indicated by a non-physiological flow pattern prompted by BMHVs [2-4]. Numerous in vivo and in vitro experimental studies have been carried out to better understand the blood flow pattern and characteristics of the design induced by BMHVs [5-9].

The experimental studies use particle image velocimetry (PIV) to compute the phase averaged flow pattern downstream of the valve leaflets. A computational study provides a resolution that is required for the mechanical environment experience due to blood cell damage and potential for

\footnotetext{
* Corresponding author.

E-mail address: mohamad.shukri@utem.edu.my (Mohamad Shukri bin Zakaria)
} 
blood clotting complications [10-16]. The BMHV flows occurring in complex geometry involve model features at disparate spatial scales, from the aorta diameter to the valve hinges and leakage jets. The pulsatile flow effects go through a transition to turbulence. In addition, the geometry of the aorta is less complicated than the left ventricle of the mitral valve so simulations in the aortic position are less definitive from computational study [1].

Factors leading to thrombosis are haemolysis and platelet activation $[17,18]$. Therefore, the risk of thrombosis can be studied by area elevated flow stresses, streamlines, recirculation zones, the dynamics of shed vortices, flow separation and turbulence [19-21]. Improving the mechanical heart valve design could reduce the risk of platelet activation and haemolysis by minimizing turbulent stresses $[17,22]$. Previous studies have supported the use of passive flow control devices such as vortex generators (VGs) on the leaflet surface to reduce turbulence and platelet activation related to the regurgitated jet $[3,23,24]$. In terms of the geometry of the VG, its shape and height could be used to overcome the flow separation problem. Studies have suggested that VGs can delay and even suppress flow separation by bringing momentum from the free stream into the boundary layer in order to provide and maintain the strong local stream wise adverse pressure gradient [23-25]. After all, the impact of VGs on BMHVs and studies concerning how to choose the appropriate VG configuration are still under investigation, especially in terms of heart valve applications.

The current study used a computational fluid dynamic (CFD) to determine the blood flow pattern in BMHVs as this software allows parametric studies to be conducted easily and economically. The objective of this study is to observe the flow around VGs in BMHVs in different models, for the axisymmetric and the anatomic aorta. The significant of the study could help to access to suitability and enhance understanding of the implications of VGs into the real anatomic patient geometry. Furthermore, to date, there are no such assessment and comparison are made between anatomic and axisymmetric aorta with VGs installed on the MHV's leaflet.

\section{Methodology}

\subsection{Geometry of the Axisymmetric Aorta and the Anatomic Aorta}

The computational model was divided into two models, namely, axisymmetric and anatomic aorta. The axisymmetric aorta was developed according to previous studies $[14,16,26,27]$. The axisymmetric aorta is considered a rigid straight tube with an inlet diameter D of $25.4 \mathrm{~mm}$ and an SJM valve diameter of $23 \mathrm{~mm}$, while the inner diameter of the valve is $21.4 \mathrm{~mm}$. The outlet diameter downstream of the valve expands to approximately $31.75 \mathrm{~mm}$. In this study, the leaflet was fixed at a fully open condition at an angle of $85^{\circ}$ along the $y-z$ plane. A general overview of an axisymmetric aorta with an SJM valve is shown in Figure 1.

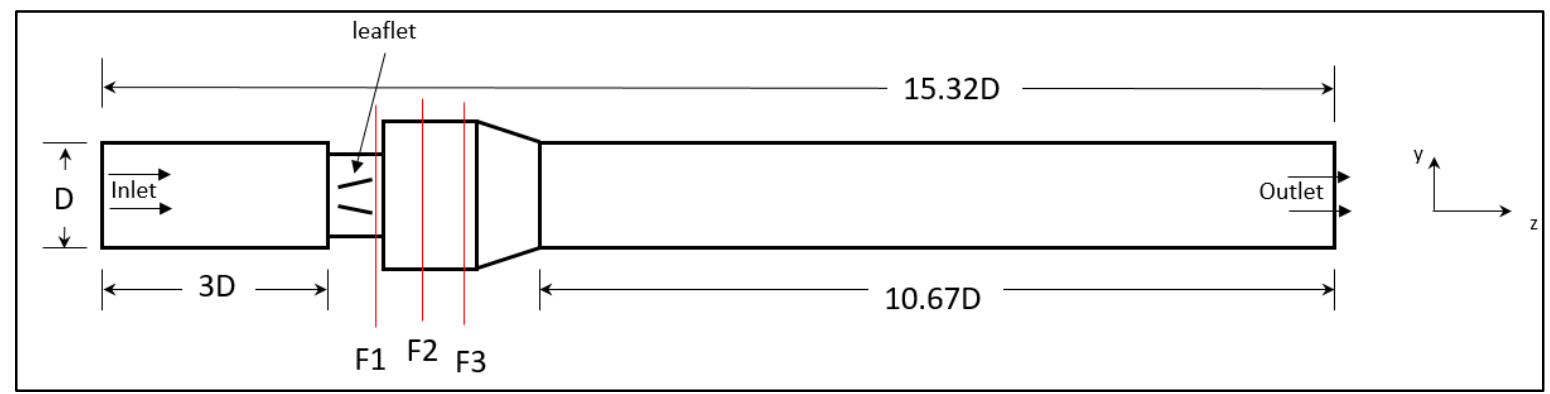

Fig. 1. The model of the axisymmetric aorta with an SJM valve

For the anatomic aorta, a CT scan was performed on a 71-year-old Malaysian male subject from the National Heart Institute Malaysia (IJN). The scan images were segmented per slice with an 
appropriate threshold value. The 3D images that contained multiple voxel data were then exported into the Stereo-lithography (STL) format. Figure 2 shows the anatomic aorta model after conversion to STL format. The methodology of converting images to CAD format has been reported in our previous work $[28,29]$.

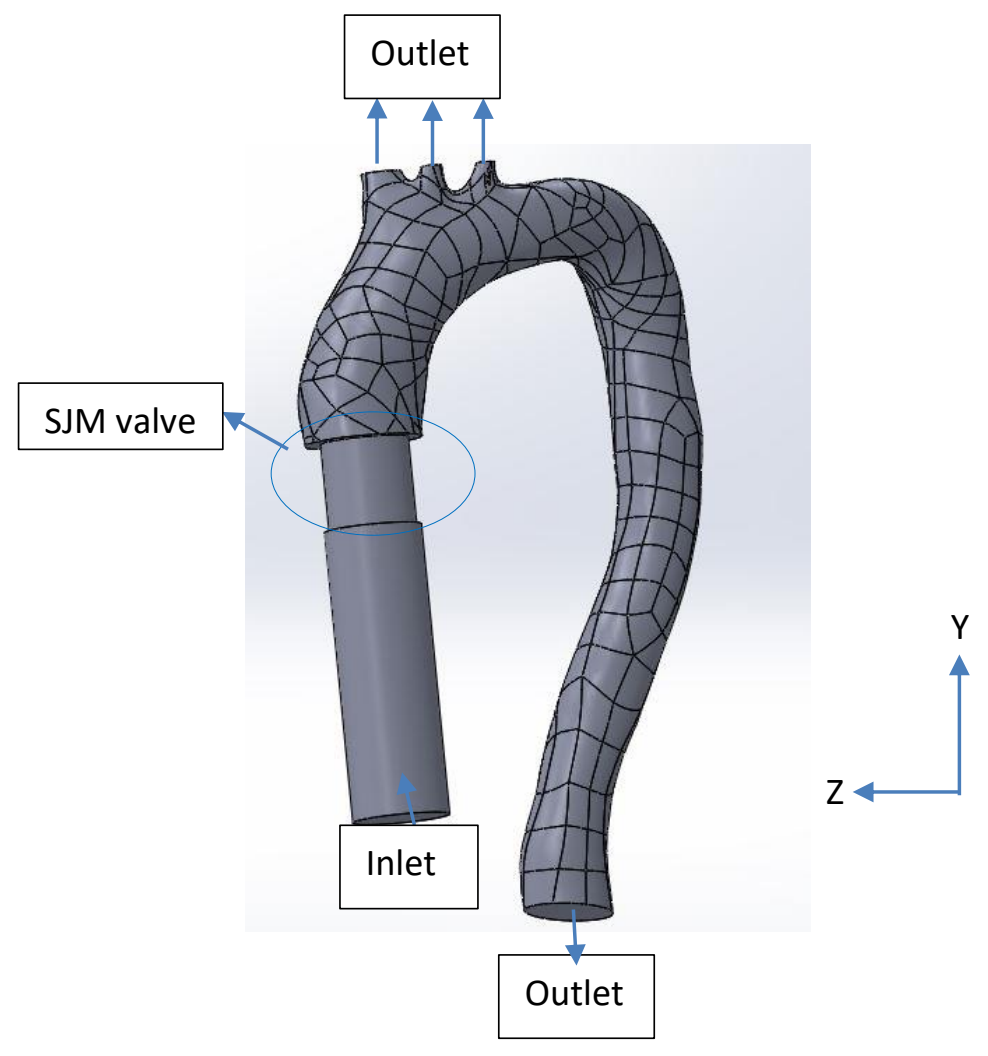

Fig. 2. Anatomic aorta after conversion to STL format with VGs

\subsection{Geometry of Vortex Generator}

VGs used in present study were triangular shape geometry as shown in Figure 3. The four VGs were mounted on the downstream side of the SJM valve leaflets with dimension of $4 \mathrm{~mm}$ length, 1 $\mathrm{mm}$ width and $1 \mathrm{~mm}$ height.

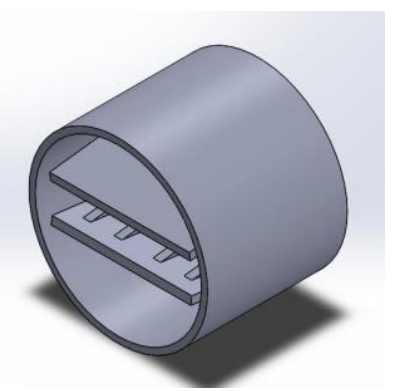

(a)

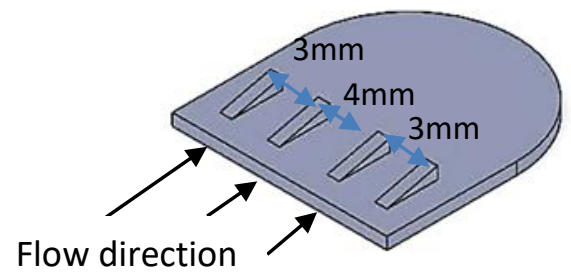

(b)

Fig. 3. VGs model (a) SJM valve with triangular VGs (b) triangular VGs with dimensions 


\subsection{Meshing}

The study comprised $400 \mathrm{~K}$ nodes and $3 \mathrm{M}$ elements that led to a skewness value of 0.84 , which is below 0.85 as suggested in Ref. [30]. Furthermore, tetrahedral meshing was considered for the axisymmetric aorta, as represented in Figure 4 . The study comprised $1 \times 10^{6}$ nodes and $3 \times 10^{6}$ elements indicated by tetrahedral and hexagonal meshing for the anatomic aorta as represented in Figure 5 . The very fine mesh made it possible to model the numerous fine curvature radii as exactly as possible and to break down turbulence as much as possible.

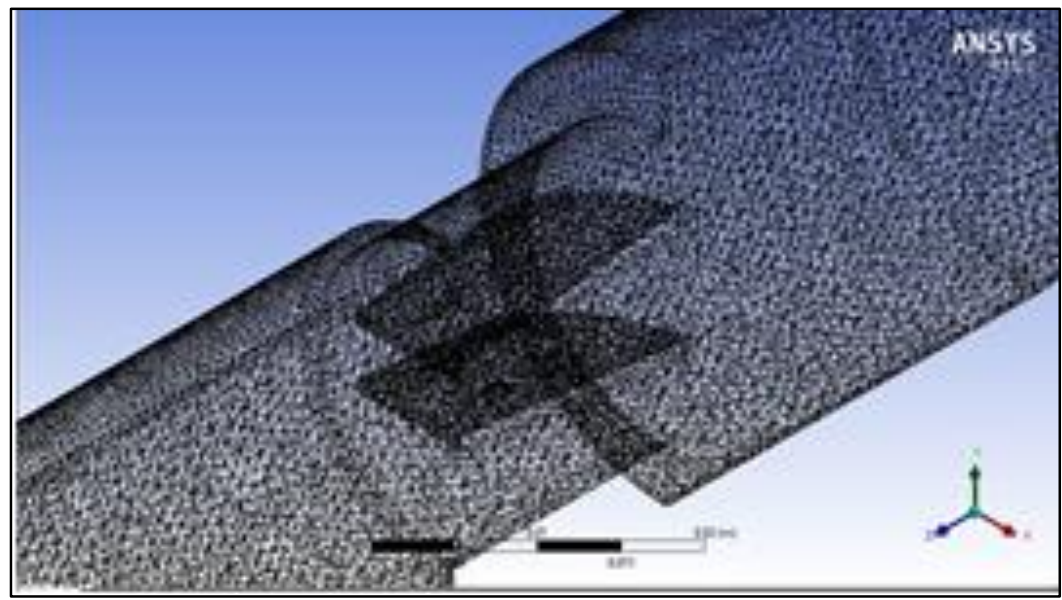

Fig. 4. The meshing configuration for the axisymmetric aorta

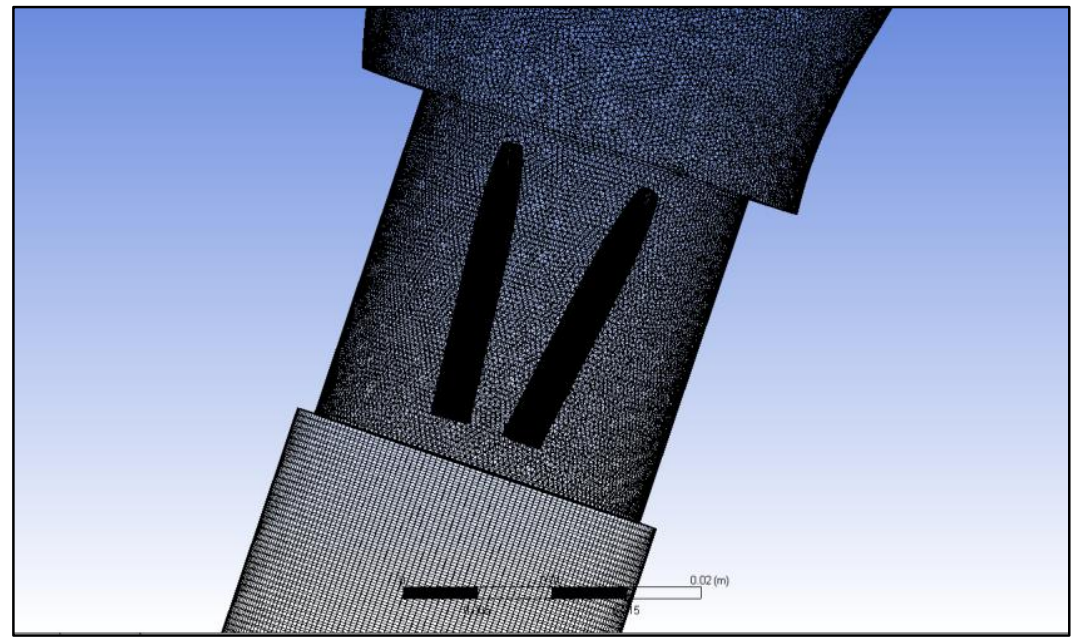

Fig. 5. The meshing configuration for the anatomic aorta

The mesh sensitivity analysis was used in the study to identify the best meshing size by saving computation time but without neglecting the accuracy of the results. The grid was tested from $4 \mathrm{x}$ $10^{5}$ until $3 \times 10^{6}$ number cells for both types of geometry model, as shown in Tables 1 and 2 respectively. The grid independency were achieved when the increase in number of cells did not affect the accuracy of the simulation results with lowest percentage of error. The grid number for both types of geometry, $1 \times 10^{6}$ to $3 \times 10^{6}$, were slightly different in their maximum velocity value at the central jet. The meshing of the $3 \mathrm{M}$ total number of elements was used for more accurate results. 
Table 1

Mesh Sensitivity analysis for the axisymmetric aorta

\begin{tabular}{lll}
\hline Element Number & Max velocity at central jet & Error \\
\hline $4 \times 10^{5}$ & 0.232 & - \\
$6 \times 10^{5}$ & 0.238 & $2.5 \%$ \\
$1 \times 10^{6}$ & 0.248 & $4.1 \%$ \\
$3 \times 10^{6}$ & 0.252 & $1.7 \%$ \\
\hline
\end{tabular}

Table 2

Mesh Sensitivity analysis for the anatomic aorta

\begin{tabular}{lll}
\hline Element Number & Max velocity at central jet & Error \\
\hline $4 \times 10^{5}$ & 0.240 & - \\
$6 \times 10^{5}$ & 0.254 & $5.51 \%$ \\
$8 \times 10^{5}$ & 0.259 & $1.93 \%$ \\
$1 \times 10^{6}$ & 0.266 & $2.63 \%$ \\
$2 \times 10^{6}$ & 0.271 & $1.84 \%$ \\
$3 \times 10^{6}$ & 0.270 & $0.37 \%$ \\
\hline
\end{tabular}

\subsection{Boundary Condition}

The inlet flows were regulated using the user-specified function comprising the centre of the $y$ plane. The pulsatile flow was assumed at the inlet conditions and the computation of the nonuniform distribution of the inlet velocity was conducted [19]. Most of the present analysis focused on three specific timings, namely accelerating flow (AF) at $t=0.1 \mathrm{~s}$, peak flow (PF) at $t=0.2 \mathrm{~s}$, and deceleration flow (DF) at $t=0.3 \mathrm{~s}$, in which all the instantaneous time was at intervals of $0.1<\mathrm{t}<0.3$ where the leaflet was fully open, as shown in Figure 6 . There was a non-slip velocity of boundary condition set along the aorta wall. The outlet boundary condition was retained at zero pascals since a similar effect is produced by fluid distribution differences concerning the flow [20].

The fluid used in the simulation had a density of $1060 \mathrm{~kg} / \mathrm{m}^{3}$ and a viscosity of $5.5 \times 10^{-03}$ with unsteady flow for $R e=6000$. The turbulent model used in this simulation was large eddy simulation (LES). A previous study used the LES model for $R e=6000$ on fixed SJM leaflets, and found that turbulent models could predict unsteady solutions with rich coherent vortex shedding [16]. In the LES turbulence model, equations were applied using Navier Stokes equations to explicitly compute large eddies. The mathematically equations were simulated by an average turbulence model between DNS and RANS in which filtered Navier Stokes equations used the following formulae:

$$
\begin{aligned}
& \frac{\partial u_{i}}{\partial t}+\frac{\partial u_{i} u_{j}}{\partial x_{j}}=-\frac{1}{\rho} \frac{\partial p}{\partial x_{i}}+\frac{\partial}{\partial x_{j}}\left(\frac{\partial u_{i}}{\partial x_{j}}\right) \\
& u_{i}(x, t)=\bar{u}_{i}(x, t)+\dot{u}_{i}(x, t) \\
& \tau_{i j}=\rho\left(\overline{u_{\imath} u_{J}}-\bar{u}_{\imath} \bar{u}_{j}\right)
\end{aligned}
$$

Combining Eq. (2) and (3) into (1), we obtain:

$$
\frac{\partial \overline{\mathrm{u}}_{\mathrm{i}}}{\partial \mathrm{t}}+\frac{\partial \overline{\mathrm{u}}_{\mathrm{i}} \overline{\mathrm{u}}_{\mathrm{j}}}{\partial \mathrm{x}_{\mathrm{j}}}=-\frac{1}{\rho} \frac{\partial \overline{\mathrm{p}}}{\partial \mathrm{x}_{\mathrm{i}}}+\frac{\partial}{\partial \mathrm{x}_{\mathrm{j}}}\left(\mathrm{v} \frac{\partial \overline{\mathrm{u}}_{\mathrm{i}}}{\partial \mathrm{x}_{\mathrm{j}}}\right)-\frac{\partial \tau_{\mathrm{ij}}}{\partial \mathrm{x}_{\mathrm{j}}}
$$


Where $u_{i}(x, t)$ is the instantaneous component, $\bar{u}_{i}(x, t)$ is the resolved scale, $\bar{u}_{\imath} \bar{u}_{j}$ is the sub grid scale and $\tau_{i j}$ is the sub grid scale turbulent stress.

Finally, the blood fluid in the simulation was assumed to be a Newtonian fluid [21]. The assumption was that the blood flow through the SJM valve was an incompressible fluid with constant density. This is because the main constituent of blood is plasma which has the properties of a Newtonian fluid in that the shear rate is linear, so this assumption was considered valid [22].

\subsection{Validation}

Figure 6 depicts the normalized velocity characteristics in the absence of a VG along a line at $x=0$ $\mathrm{mm}$. The tips leaflet (F1) and the downstream valves (F2 and F3) of the axisymmetric aorta were evaluated to determine the velocity profile of blood, and the results were used for validation. The velocity profile specified in previous research [16] comprised similar flow characteristics and geometry concerning the valve. The results obtained from previous research and the present study are in line with those suggested by computational information. The root mean square (RMS) velocity difference was computed to determine the difference between the values in previous studies and the present study. The computed difference in RMS velocity was only $1.4 \%$ of the maximum velocity, indicating agreement between the study results and the calculated values.
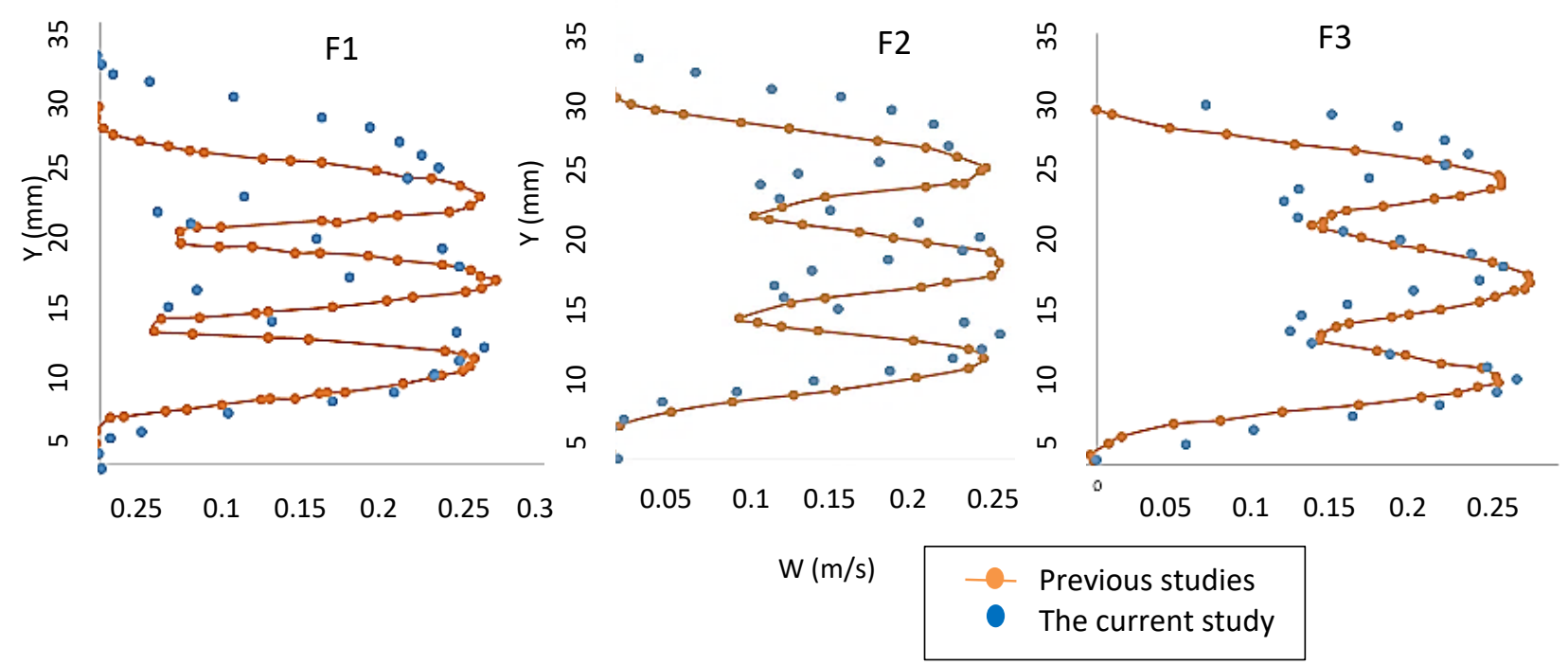

Fig. 6. The comparison of velocity profile of F1, F2 and F3 [16]

\section{Result and Discussion}

Figure 7 shows the comparison of the velocity magnitude for acceleration flow (AF), peak flow (PF) and deceleration flow (DF). The central jet (between the leaflets) did not change significantly at the AF phase, where the velocity range for both models was between 0.65 and $0.75 \mathrm{~m} / \mathrm{s}$. However, it started to increase at the PF phase when the two lateral (between the aorta wall and the leaflets) and central jets continued to produce uniform velocity for the axisymmetric aorta compared to the anatomic aorta. At the DF phase, it was noted that the central jet for the anatomic aorta $(1.2 \mathrm{~m} / \mathrm{s})$ increased more than for the axisymmetric aorta $(0.8 \mathrm{~m} / \mathrm{s})$. This happened due to the area expansion in the sinus region. Besides that, the anatomic aorta curvature induced a strong secondary motion 
that produced a lower velocity at the inner bend compared to the outer bend and therefore produced higher wall shear stress that contributed to the potential for more blood clotting [31].

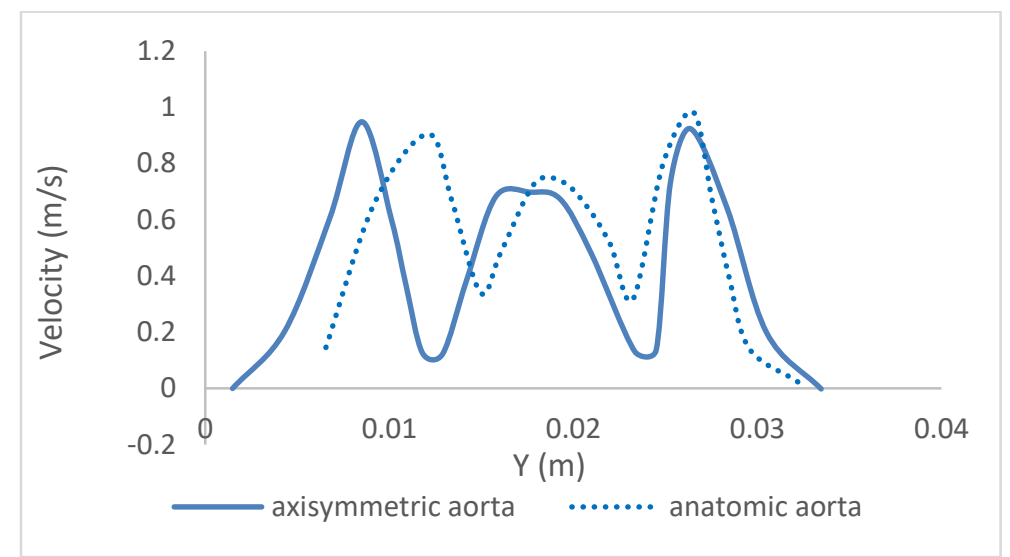

(a)

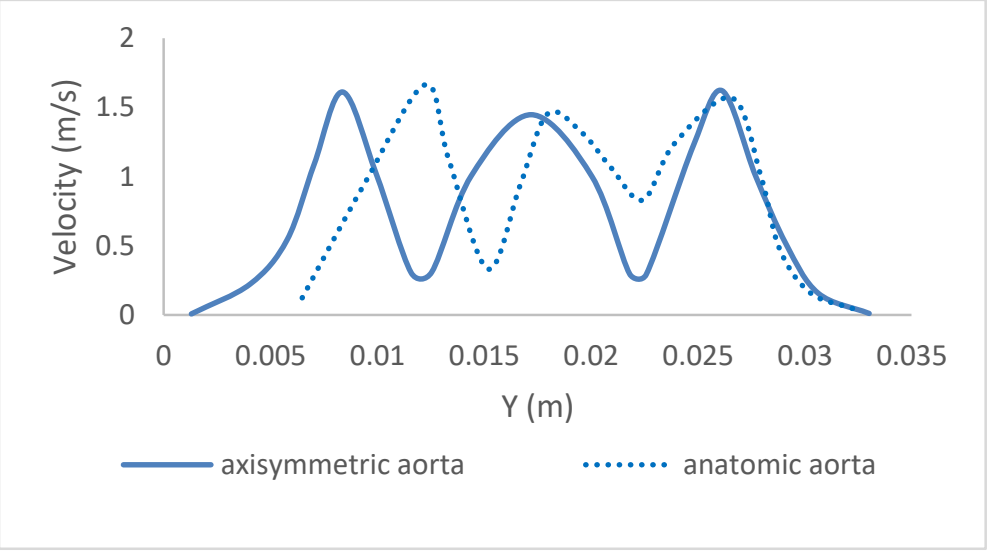

(b)

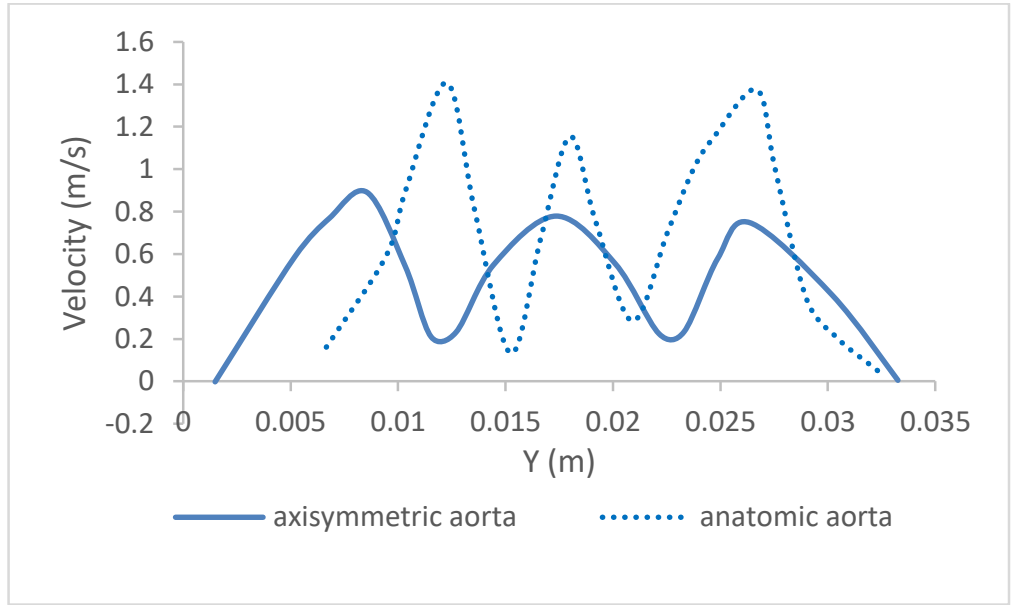

(c)

Fig. 7. The velocity profile comparison between the axisymmetric and the anatomic aorta at three phases: (a) AF (b) PF (c) DF

The velocity vectors in Figure 8 were used to oversee the recirculation areas through the axisymmetric and anatomic aorta, which are marked with circles. The recirculation regions indicate the platelets that became trapped and later increased in residence time, thus increasing the potential for blood clotting. Observations were conducted of the recirculation region at the aortic root region and the wake of the leaflets for the axisymmetric and anatomic aorta. When the area expanded with 
time, most of the recirculation zone locations were unchanged. The behaviour continued until the decelerating flow. The difference between the axisymmetric and anatomic aorta models was that more circulation existed after the sinus wall.

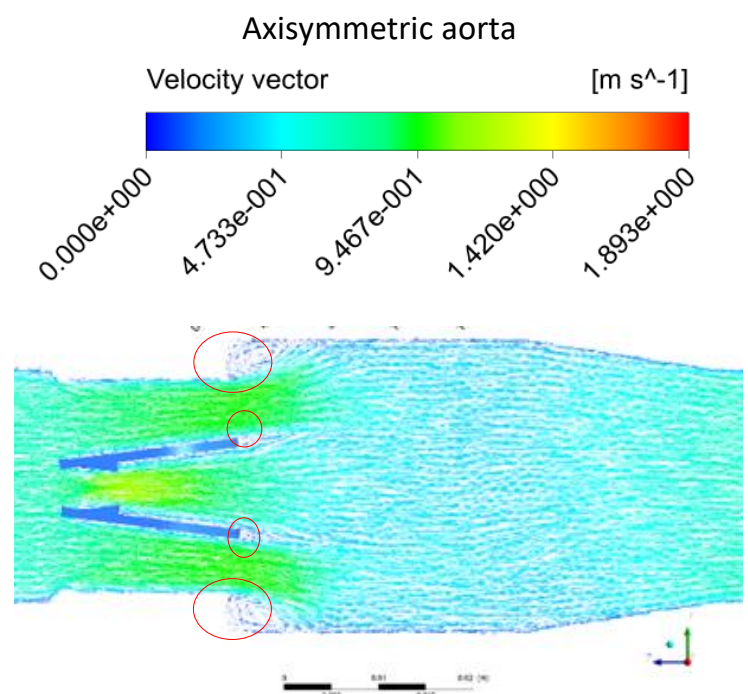

(a)

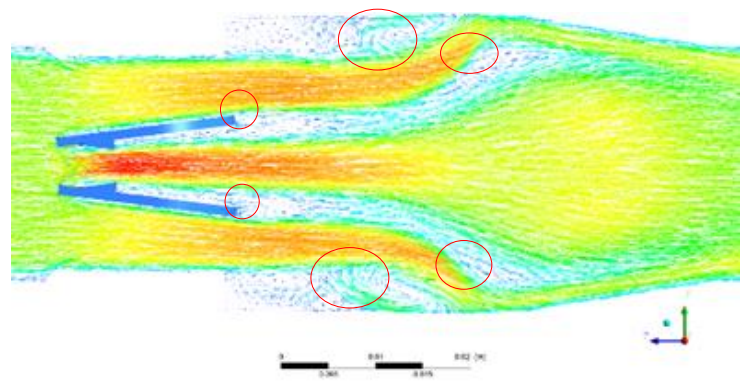

(b)

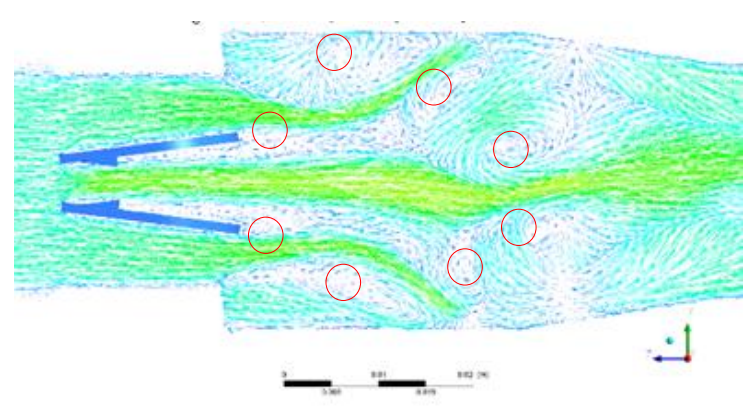

(c)
Anatomic aorta

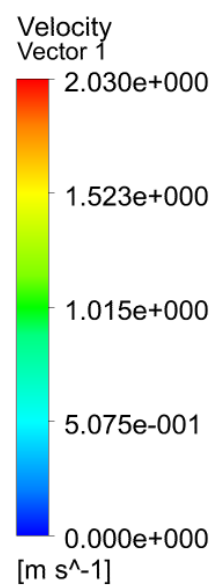

$(d)$

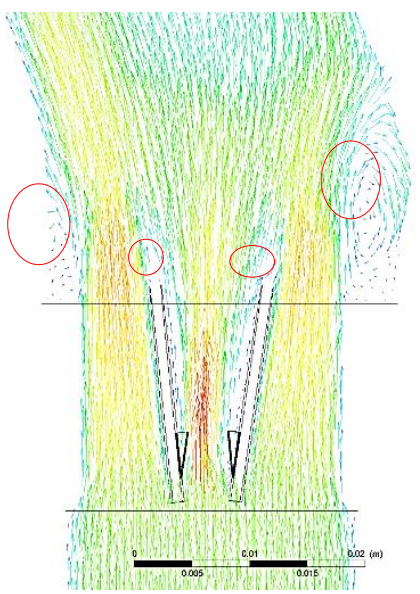

(e)

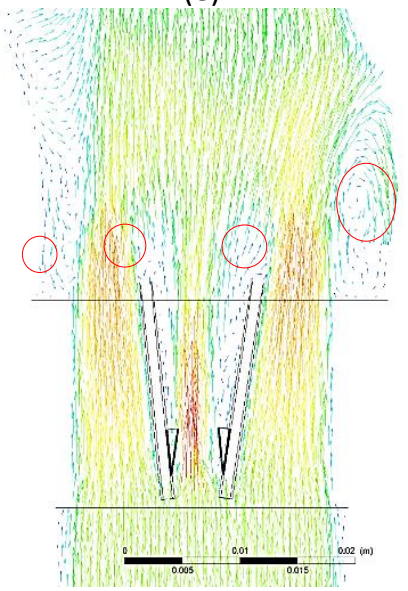

(f)

Fig. 8. Velocity vector field of the SJM heart valve: (a) axisymmetric aorta, AF (b) axisymmetric aorta, PF (c) axisymmetric aorta, DF (d) anatomic aorta, AF (e) anatomic aorta, PF (f) anatomic aorta, DF 
Figure 9 shows the wall shear stress (WSS) on the SJM valve frame at the axisymmetric aorta. Lower WSS was found at the AF phase. WSS increased once the PF phase was reached when it passed through the valve frame. High WSS developed around the valve frame, indicating the development of thrombosis [32]. Figure 10 shows the WSS on the SIM valve frame attached to the anatomic aorta. It shows the maximum values of WSS applied to the anatomic aorta occurred at the PF phase. The anatomic aorta WSS was not significantly changed as the flow phase increased. Comparing between these two models, anatomic aorta experience higher WSS indicating high risk of thrombosis potential.

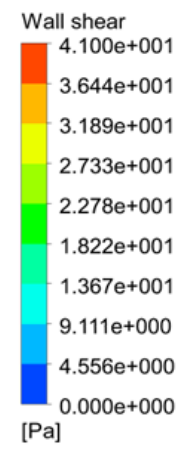

(a)

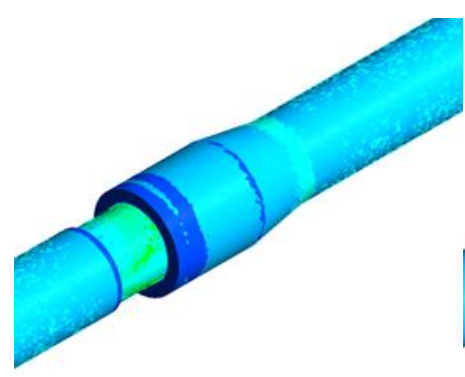

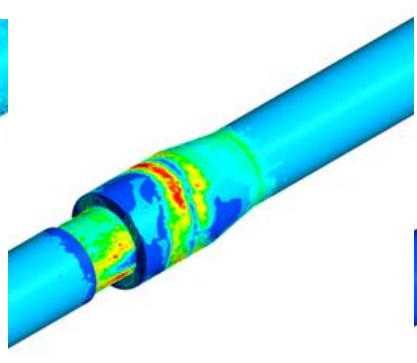

(b)

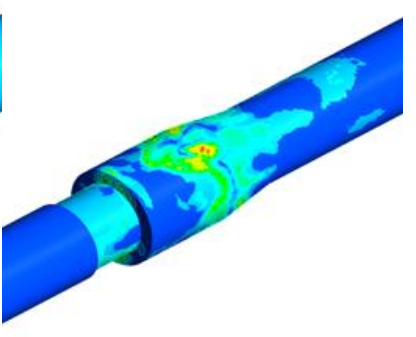

(c)

Fig. 9. WSS of SJM heart valve: (a) axisymmetric aorta, AF (b) axisymmetric aorta, PF (c) axisymmetric aorta, DF

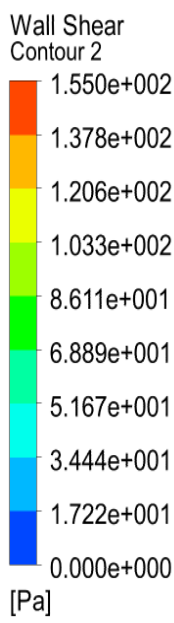

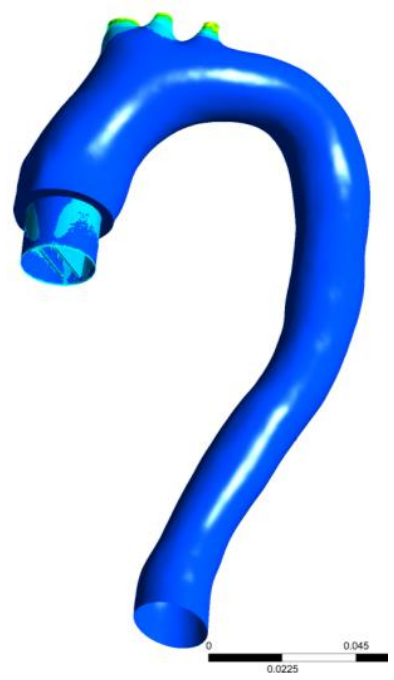

(a)

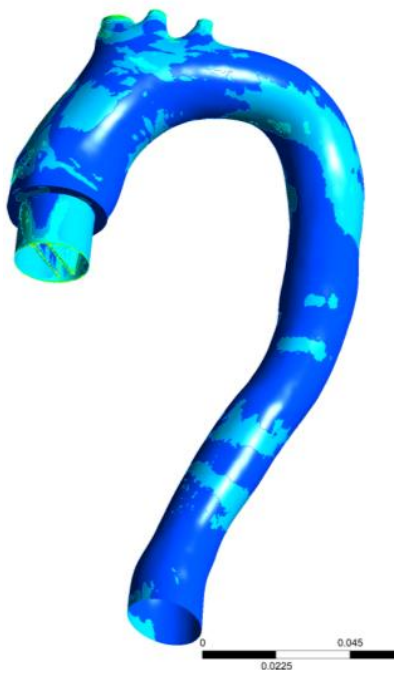

(b)

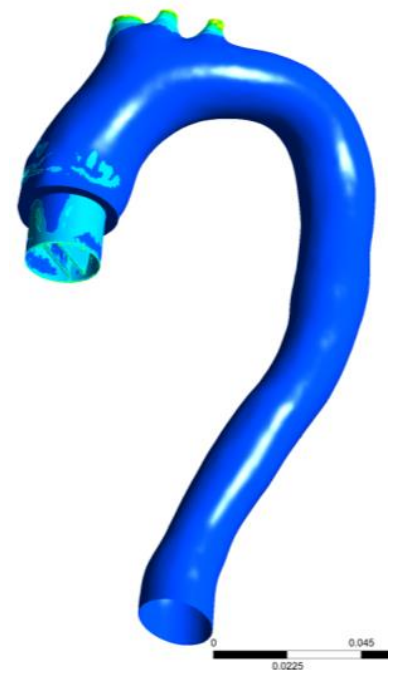

(c)

Fig. 10. WSS of SJM heart valve: (a) anatomic aorta, AF (b) anatomic aorta, PF (c) anatomic aorta, DF

\section{Conclusions}

In this study, blood flow through the SJM valve was analysed for the axisymmetric aorta and the anatomic aorta. A design process including VGs on the leaflet surface and the use of modelled anatomic aorta would allow the predicted flow field parameter to be estimated for a real human body. Result indicates that the model show maximum velocity magnitude with $1.83 \mathrm{~m} / \mathrm{s}$ (axisymmetric aorta) and $2.03 \mathrm{~m} / \mathrm{s}$ (anatomic aorta) at the PF phase. The axisymmetric aorta tends to reduce blood clotting since it has lower WSS with 28Pa. The WSS for anatomic aorta has high WSS 
with 69Pa that will developed more thrombosis. Results also suggested that using different forms of geometry could affect the blood flow hemodynamic characteristics, and the flow patterns. The simulation data demonstrates that an additional location of blood clotting was found at the outer curvature of the aorta. Furthermore, the anatomic aorta is prone to develop more blood clots as a result of higher velocity and lower WSS, nevertheless the use of passive flow control such as VG.

\section{Acknowledgement}

The authors would like to thank the Universiti Teknikal Malaysia Melaka (UTeM) and the Ministry Education Malaysia (MOE) for funding this research project through grant no: FRGS/2018/FKMCARE/F00367, and the Faculty of Mechanical Engineering at UTeM for its practical support. The authors also thank to National Heart Insitute, Malaysia (IJN), for their co-operation in fulfilling the requirement of this study through our co-author (adiazriff@upm.edu.my).

\section{References}

[1] Sotiropoulos, Fotis, and Iman Borazjani. "A review of state-of-the-art numerical methods for simulating flow through mechanical heart valves." Medical \& biological engineering \& computing 47, no. 3 (2009): 245-256. https://doi.org/10.1007/s11517-009-0438-z

[2] Bellhouse, B. J. "Fluid mechanics of a model mitral valve and left ventricle." Cardiovascular research 6, no. 2 (1972): 199-210. https://doi.org/10.1093/cvr/6.2.199

[3] Ge, Liang, Lakshmi P. Dasi, Fotis Sotiropoulos, and Ajit P. Yoganathan. "Characterization of hemodynamic forces induced by mechanical heart valves: Reynolds vs. viscous stresses." Annals of Biomedical Engineering 36, no. 2 (2008): 276-297. https://doi.org/10.1007/s10439-007-9411-x

[4] Yin, Wei, Yared Alemu, Klaus Affeld, Jolyon Jesty, and Danny Bluestein. "Flow-induced platelet activation in bileaflet and monoleaflet mechanical heart valves." Annals of biomedical engineering 32, no. 8 (2004): 1058-1066. https://doi.org/10.1114/b:abme.0000036642.21895.3f

[5] Dasi, L. P., L. Ge, H. A. Simon, Fotis Sotiropoulos, and A. P. Yoganathan. "Vorticity dynamics of a bileaflet mechanical heart valve in an axisymmetric aorta." Physics of Fluids 19, no. 6 (2007): 067105. https://doi.org/10.1063/1.2743261

[6] Ellis, Jeffrey T., Timothy M. Healy, Arnold Anthony Fontaine, Rahul Saxena, and Ajit P. Yoganathan. "Velocity measurements and flow patterns within the hinge region of a Medtronic Parallel bileaflet mechanical valve with clear housing." The Journal of heart valve disease 5, no. 6 (1996): 591-599. https://doi.org/10.1097/00002480$199703000-00085$

[7] Feng, Zhonggang, Mitsuo Umezu, Tetsuo Fujimoto, Toshiya Tsukahara, Masakazu Nurishi, and Daisuke Kawaguchi. "In vitro hydrodynamic characteristics among three bileaflet valves in the mitral position." Artificial organs 24 , no. 5 (2000): 346-354. https://doi.org/10.1046/i.1525-1594.2000.06498.x

[8] Ge, Liang, and Fotis Sotiropoulos. "A numerical method for solving the 3D unsteady incompressible Navier-Stokes equations in curvilinear domains with complex immersed boundaries." Journal of computational physics 225, no. 2 (2007): 1782-1809. https://doi.org/10.1016/i.jcp.2007.02.017

[9] Travis, Brandon R., Hwa L. Leo, Parina A. Shah, David H. Frakes, and Ajit P. Yoganathan. "An analysis of turbulent shear stresses in leakage flow through a bileaflet mechanical prostheses." J. Biomech. Eng. 124, no. 2 (2002): 155165. https://doi.org/10.1115/1.1448519

[10] Zakaria, Mohamad Shukri, Farzad Ismail, Masaaki Tamagawa, Ahmad Fazli Abdul Azi, Surjatin Wiriadidjaya, Adi Azrif Basri, and Kamarul Arifin Ahmad. "Computational fluid dynamics study of blood flow in aorta using OpenFOAM." Journal of Advanced Research in Fluid Mechanics and Thermal Sciences 43, no. 1 (2018): 81-89.

[11] Zakaria, Mohamad Shukri, Farzad Ismail, Masaaki Tamagawa, Ahmad Fazli Abdul Aziz, Surjatin Wiriadidjaja, Adi Azrif Basri, and Kamarul Arifin Ahmad. "Review of numerical methods for simulation of mechanical heart valves and the potential for blood clotting." Medical \& biological engineering \& computing 55, no. 9 (2017): 1519-1548. https://doi.org/10.1007/s11517-017-1688-9

[12] Salleh, Nursyaira Mohd, Mohamad Shukri Zakaria, and Mohd Juzaila Abd Latif. "The vortex shedding for an inclined flat plate of thrombosis using CFD simulation." Proceedings of Mechanical Engineering Research Day 20192019 (2019): 152-154.

[13] Salleh, Nursyaira Mohd, Mohamad Shukri Zakaria, and Mohd Juzaila Abd Latif. "Reducing of Thrombosis in Mechanical Heart Valve through the Computational Method: A Review." Journal of Advanced Research in Fluid Mechanics and Thermal Sciences 65, no. 2 (2020): 178-200. 
[14] Yun, B. Min, L. P. Dasi, C. K. Aidun, and A. P. Yoganathan. "Computational modelling of flow through prosthetic heart valves using the entropic lattice-Boltzmann method." Journal of Fluid Mechanics 743 (2014): 170. https://doi.org/10.1017/ifm.2014.54

[15] Yun, B. Min, L. P. Dasi, C. K. Aidun, and A. P. Yoganathan. "Highly resolved pulsatile flows through prosthetic heart valves using the entropic lattice-Boltzmann method." Journal of Fluid Mechanics 754 (2014): 122. https://doi.org/10.1017/ifm.2014.393

[16] Ge, Liang, Hwa-Liang Leo, Fotis Sotiropoulos, and Ajit P. Yoganathan. "Flow in a mechanical bileaflet heart valve at laminar and near-peak systole flow rates: CFD simulations and experiments." (2005): $782-797$. https://doi.org/10.1115/1.1993665

[17] Dasi, Lakshmi P., Helene A. Simon, Philippe Sucosky, and Ajit P. Yoganathan. "Fluid mechanics of artificial heart valves." Clinical and experimental pharmacology and physiology 36, no. 2 (2009): $225-237$. https://doi.org/10.1111/i.1440-1681.2008.05099.x

[18] Hatoum, Hoda, Atieh Yousefi, Scott Lilly, Pablo Maureira, Juan Crestanello, and Lakshmi P. Dasi. "An in vitro evaluation of turbulence after transcatheter aortic valve implantation." The Journal of thoracic and cardiovascular surgery 156, no. 5 (2018): 1837-1848. https://doi.org/10.1016/i.jtcvs.2018.05.042

[19] Bluestein, Danny, Edmond Rambod, and Morteza Gharib. "Vortex shedding as a mechanism for free emboli formation in mechanical heart valves." J. Biomech. Eng. 122, no. 2 (2000): 125-134. https://doi.org/10.1115/1.429634

[20] Chandran, Krishnan B. "Role of computational simulations in heart valve dynamics and design of valvular prostheses." Cardiovascular engineering and technology 1, no. 1 (2010): 18-38. https://doi.org/10.1007/s13239010-0002-X

[21] Hatoum, Hoda, and Lakshmi P. Dasi. "Reduction of pressure gradient and turbulence using vortex generators in prosthetic heart valves." Annals of biomedical engineering 47, no. 1 (2019): 85-96. https://doi.org/10.1007/s10439-018-02128-6

[22] Zilla, Peter, Johan Brink, Paul Human, and Deon Bezuidenhout. "Prosthetic heart valves: catering for the few." Biomaterials 29, no. 4 (2008): 385-406. https://doi.org/10.1016/j.biomaterials.2007.09.033

[23] Lin, Chou Aw, Fatimah Al-Zahrah Mohd Sa'at, Fadhilah Shikh Anuar, Mohamad Firdaus Sukri, Mohd Zaid Akop, and Zainuddin Abdul Manan. "Heat Transfer Across Tube Banks With a Passive Control Vortex Generator in Steady OneDirectional and Oscillatory Flows." CFD Letters 13, no. 1 (2021): 1-18. https://doi.org/10.37934/cfdl.13.1.118

[24] Mugisidi, Dan, Oktarina Heriyani, Pancatatva Hesti Gunawan, and Dwi Apriani. "Performance Improvement of a Forced Draught Cooling Tower Using a Vortex Generator." CFD Letters 13, no. 1 (2021): 45-57. https://doi.org/10.37934/cfdl.13.1.4557

[25] Godard, Gilles, and Michel Stanislas. "Control of a decelerating boundary layer. Part 1: Optimization of passive vortex generators." Aerospace Science and Technology 10, no. 3 (2006): $181-191$. https://doi.org/10.1016/j.ast.2005.11.007

[26] Dasi, Lakshmi P., David W. Murphy, Ari Glezer, and Ajit P. Yoganathan. "Passive flow control of bileaflet mechanical heart valve leakage flow." Journal of Biomechanics 41, no. $6 \quad$ (2008): 1166-1173. https://doi.org/10.1016/i.jbiomech.2008.01.024

[27] Murphy, David W., Lakshmi P. Dasi, Jelena Vukasinovic, Ari Glezer, and Ajit P. Yoganathan. "Reduction of procoagulant potential of b-datum leakage jet flow in bileaflet mechanical heart valves via application of vortex generator arrays." Journal of biomechanical engineering 132, no. 7 (2010). https://doi.org/10.1115/1.4001260

[28] Basri, Adi A., Mohammad Zuber, Ernnie I. Basri, Muhammad S. Zakaria, Ahmad FA Aziz, Masaaki Tamagawa, and Kamarul A. Ahmad. "Fluid Structure Interaction on Paravalvular Leakage of Transcatheter Aortic Valve Implantation Related to Aortic Stenosis: A Patient-Specific Case." Computational and mathematical methods in medicine 2020 (2020). https://doi.org/10.1155/2020/9163085

[29] Lim, Sheh Hong, Mohd Azrul Hisham Mohd Adib, Mohd Shafie Abdullah, Nur Hartini Mohd Taib, Radhiana Hassan, and Azian Abd Aziz. "Study of Extracted Geometry Effect on Patient-Specific Cerebral Aneurysm Model with Different Threshold Coefficient (Cthres)." CFD $\quad$ Letters 12, $\quad$ no. $10 \quad$ (2020): 1-14. https://doi.org/10.37934/cfdl.12.10.114

[30] Chirica, I., C. M. Angheluta, S. D. Perijoc, A. I. Hobjilă, and M. Frătita. "Mesh independence of a transient multiphase fluid-solid interaction." In Journal of Physics: Conference Series, vol. 1297, no. 1, p. 012026. IOP Publishing, 2019. https://doi.org/10.1088/1742-6596/1297/1/012026

[31] Khalili, Fardin, Peshala Gamage, and Hansen A. Mansy. "Hemodynamics of a bileaflet mechanical heart valve with different levels of dysfunction." arXiv preprint arXiv:1711.11153 (2017). https://doi.org/10.15406/jabb.2017.02.00044

[32] Shipkowitz, Tanya, Jeffrey Ambrus, James Kurk, and Kosala Wickramasinghe. "Evaluation technique for bileaflet mechanical valves." The Journal of heart valve disease 11, no. 2 (2002): 275-282. https://doi.org/10.1097/00002480-199903000-00051 